\title{
Extending the Fungal Host Range of a Partitivirus and a Mycoreovirus from Rosellinia necatrix by Inoculation of Protoplasts with Virus Particles
}

\author{
Satoko Kanematsu, Atsuko Sasaki, Mari Onoue, Yuri Oikawa, and Tsutae Ito
}

First, third, fourth, and fifth author: Apple Research Station, National Institute of Fruit Tree Science, NARO, Shimokuriyagawa, Morioka 020-0123, Japan; and second author: National Institute of Fruit Tree Science, NARO, Fujimoto 2-1, Tsukuba 305-8605, Japan. Accepted for publication 10 May 2010.

\begin{abstract}
Kanematsu, S., Sasaki, A., Onoue, M., Oikawa, Y., and Ito, T. 2010. Extending the fungal host range of a partitivirus and a mycoreovirus from Rosellinia necatrix by inoculation of protoplasts with virus particles. Phytopathology 100:922-930.

The potential host range of mycoviruses is poorly understood because of the lack of suitable inoculation methods. Recently, successful transfection has been reported for somatically incompatible fungal isolates with purified virus particles of two mycoviruses, the partitivirus RnPV1-W8 (RnPV1) and the mycoreovirus RnMyRV3/W370 (MyRV3), from the white root rot fungus Rosellinia necatrix (class Sordariomycetes, subclass Xylariomycetidae). These studies examined and revealed the effect of the mycoviruses on growth and pathogenicity of $R$. necatrix. Here, we extended the experimental host range of these two mycoviruses using a transfection approach. Protoplasts of other phytopathogenic Sordariomycetous fungi-Diaporthe sp., Cryphonectria parasitica, Valsa ceratosperma (Sordariomycetidae), and Glomerella
\end{abstract}

ABSTRACT

Mycovirus infections are thought to be common in all major taxonomic groups of filamentous fungi. Many mycoviruses have spherical virus particles with double-stranded (ds)RNA genomes. Most mycoviruses with dsRNA genome are classified into four families: Partitiviridae, Totiviridae, Chyrysoviridae, and Reoviridae. Single-stranded (ss)RNA mycoviruses with or without coat proteins are classified into five families, while many other viruses remain unassigned (13). Although most mycoviruses have no overt effect on their fungal hosts, particular mycoviruses appear to have significant phenotypic effects, including a reduction in the virulence of phytopathogenic fungal hosts, referred to as hypovirulence. Such mycoviruses (hypovirulence factors) are expected to be used as biocontrol agents and as tools to elucidate the mechanism of fungal pathogenicity (13).

Rosellinia necatrix Berl. ex Prill. is a soilborne ascomycete belonging to Sordariomycetes that causes white root rot in a wide range of perennial plants. White root rot disease leads to serious reductions in fruit tree production of Japanese pear, apple, and grape in Japan $(9,18)$ and the avocado in Spain $(32)$. R. necatrix propagates mainly through mycelial growth in the soil and has a simple population structure within individual orchards $(\mathrm{H}$. Nakamura, personal communication). Thus, we are exploring the use of a mycovirus for the biological control of $R$. necatrix (24) because the less diverse population would enhance mycovirus

Corresponding author: S. Kanematsu; E-mail address: satokok@affrc.go.jp

doi:10.1094/PHYTO-100-9-0922

(C) 2010 The American Phytopathological Society cingulata (Hypocreomycetidae)—were inoculated with RnPV1 and MyRV3 viral particles. The presence of double-stranded RNA viral genomes in regenerated mycelia of Diaporthe sp., C. parasitica, and $V$. ceratosperma confirmed both types of viral infections in these three novel host species. An established RnPV1 infection was confirmed in $G$. cingulata but MyRV3 did not infect this host. Horizontal transmission of both viruses from newly infected strains to virus-free, wild-type strains through hyphal anastomosis was readily achieved by dual culture; however, vertical transmission through conidia was rarely observed. The virulence of Diaporthe sp., C. parasitica, and V. ceratosperma strains harboring MyRV3 was reduced compared with their virus-free counterpart. In summary, our protoplast inoculation method extended the experimental host range of RnPV1-W8 and MyRV3 within the class Sordariomycetes and revealed that MyRV3 confers hypovirulence to the new hosts, as it does to R. necatrix.

Additional keywords: biological control, virocontrol. transmission via hyphal anastomosis within an individual orchard, once mycoviruses were introduced. More than 1,000 isolates of $R$. necatrix have been collected throughout Japan for identification of hypovirulence factors, and an estimated $20 \%$ of these fungal isolates contain diverse dsRNAs which were assumed to be mycovirus genomes $(2,16)$. Two of these virus-containing isolates, W8 and W370, showed irregular colony morphology and low virulence.

R. necatrix W8 harbors four dsRNAs (L1, L2, M1, and M2, named according to size). The $\mathrm{M}$ dsRNAs were identified as the genome of partitivirus Rosellinia necatrix partitivirus 1-W8 (RnPV1-W8) while the L dsRNAs are thought to belong to a distinct virus (35). RnPV1-W8 has isometric particles with an diameter of $\approx 25 \mathrm{~nm}$ comprising two genomic dsRNAs (2,299 and $2,279 \mathrm{bp}$ ) (35). Using protoplast transfection of purified virus particles, Sasaki et al. (34) demonstrated directly that single infection with RnPV1-W8 has no overt effect on mycelial growth or pathogenicity of $R$. necatrix. This result supports the general view that most partitivirus are cryptically present within a wide variety of hosts, such as ascomycetes and basidiomycetes (12).

In contrast, $R$. necatrix isolate W370 harbored double-shelled, spherical particles $80 \mathrm{~nm}$ in diameter comprising equimolar amounts of 12 segmented genomic dsRNAs of 943 to 4,143 bp $(31,43,44)$. Morphology of these virus particles and analysis of its genome sequence indicated that the virus is a novel reovirus designated as Rosellinia necatrix Mycoreovirus 3 or RnMyRV3/ W370 (MyRV3) $(31,43,44)$. MyRV3, along with two Cryphonectria parasitica Mycoreovirus spp. (MyRV1 and MyRV2), belong to a newly established genus Mycoreovirus in the family 
Reoviridae (28). MyRV3 infection alters colony morphology and reduces the virulence of host fungus $R$. necatrix (19); similarly, MyRV1 and MyRV2 infections cause hypovirulence in $C$. parasititca $(10,14)$.

The natural host ranges of mycoviruses are thought to be limited to a single species or to very closely related species because the only proven route of mycovirus horizontal transmission in filamentous fungi is via hyphal fusion. The host range of a hypovirus in the family Hypoviridae has been experimentally extended $(4,36)$. Hypoviruses are RNA viruses which are not associated with rigid virus particles, and their associated dsRNAs are now thought to be the replicative intermediate of ssRNA genomes, like that of single-strand, positive sense RNA mycoviruses (13). Using viral cDNA or synthetic transcripts, the prototype hypovirus CHV-EP713 was shown to infect fungal species within the same fungal order to which its natural host belong and to reduce virulence of the infected hosts $(4,36)$. By contrast, expanding the experimental host range of dsRNA mycoviruses with rigid virus particles has not been investigated because inoculation of fungal hosts with dsRNA mycoviruses was once regarded as difficult (13). Recently, stable infection systems with rigid virus particles have been reported for some dsRNA mycoviruses in families Mycoreoviridae $(14,33)$ and Partitiviridae (34). These promising infection systems could be applicable to inoculation of other fungal genera with virus particles for investigation of the potential, experimental host range of other mycoviruses.

We examined the infectivity of partitivirus RnPV1 and mycoreovirus MyRV3, both isolated from $R$. necatrix, in different fungal hosts within Sordariomycetes using PEG-mediated protoplast transfection with virus particles as the mechanism of infection. "Transfection" is defined as inoculation of fungal protoplasts with virus particles (14). These viruses were shown to be transmitted from transfectants to virus-free parent strains. We examined and characterized the effect of these infections on colony morphology and pathogenicity of novel fungal host species.

\section{MATERIALS AND METHODS}

Fungal isolates and culture conditions. $R$. necatrix isolate W8 contained four dsRNAs, the two genome dsRNAs of RnPV1-W8 (35) and the L1 and L2 dsRNAs. The two genomic dsRNA segments of RnPV1-W8 were almost the same size (2,299 and 2,279 bp) and, therefore, co-migrated as one band when analyzed by agarose gel electrophoresis (35). Virus-free isolate W97 was inoculated with purified RnPV1 particles from isolate W8, as described by Sasaki et al. (34), to obtain strain W97(RnPV1)-1. RnPV1 virus particles were reisolated from strain W97(RnPV1)-1 and used to inoculate isolate W97. We repeated three cycles of this reisolation-transfection procedure in order to eliminate contamination of W97(RnPV1) by L1 or L2 dsRNA, and the final strain W97(RnPV1)-3 was used to prepare the RnPV1-W8 particles used in this study. R. necatrix isolate W370 (19) was used to isolate MyRV3-RnW370 particles. Mycelia used to isolate RnPV1 and MyRV3 particles were cultured in Czapek-Dox broth and on cellophane-overlaid potato dextrose agar (PDA) (Becton Dickinson, Sparks, MD), respectively, and samples were stored at -30 or $-80^{\circ} \mathrm{C}$.

Four potential fungal hosts-Diaporthe sp. G-type 3a (21), $C$. parasitica 155/2 (36), Valsa ceratosperma AVC53 (36), and Glomerella cingulata 046-71 (from a diseased apple provided by Y. Iwanami, Nagano Fruit Tree Research Station, Japan)—were used for inoculation with virus particles. All the fungal strains were cultured on PDA and maintained at $5^{\circ} \mathrm{C}$ until use or kept in $10 \%$ glycerol stock at $-80^{\circ} \mathrm{C}$ for long-term storage. For dsRNA extraction, mycelia were cultured in potato dextrose broth (PDB) (Becton Dickinson). Mycelia used for protoplast preparation were obtained as follows. Conidia grown on PDA plates or homo- genized mycelia were suspended in PDB and cultured overnight at $25^{\circ} \mathrm{C}$ with gentle shaking. PDB cultures containing germinated conidia or the homogenized mycelia were transferred to complete medium (CM) broth (22). CM broth cultures were incubated for 24 to $36 \mathrm{~h}$ at 25 to $26^{\circ} \mathrm{C}$ with gentle shaking. Mycelia were collected by centrifugation at $5,000 \times g$ for 5 to $10 \mathrm{~min}$.

Northern analysis. Northern hybridization was performed to confirm the absence of L1 and L2 dsRNAs in strain W97(RnPV1)-3. L1 and L2 dsRNAs in W8 were fractionated in $0.7 \%$ agarose gel, cut out separately, and extracted with the RNaid KIT (BIO 101 Inc., LaJolla, CA). Probes were prepared from each dsRNA segment with AlkPhos Direct (GE Healthcare, Tokyo) as described in Ikeda et al. (16). Digoxigenin-labeled RNA-dependent RNA polymerase (RdRp) and coat protein $(\mathrm{CP})$ probes of RnPV1 were prepared, using T7 and SP6 primers from pGEM-T vector that contained the entire sequence of each segment, according to the manufacturer's recommendation (Roche, Tokyo). Northern hybridization and signal detection were performed as described previously (34).

Purification of viral particles. Virus particles of RnPV1-W8 were purified from strain W97(RnPV1)-3 as described previously (34). Particles of MyRV3 were obtained from strain W370 as follows. Mycelia (3 $\mathrm{g}$, fresh weight) were ground in a mortar with a pestle under liquid nitrogen. Powdered mycelia were transferred into TN buffer (20 mM Tris- $\mathrm{HCl}[\mathrm{pH} 7.4]$ and $0.4 \mathrm{M} \mathrm{NaCl})$ (29) containing $0.1 \%$ 2-mercaptoethanol and homogenized in a Waring blender at $13,000 \mathrm{rpm}$ for $10 \mathrm{~min}$. Mycelial suspensions were clarified twice with 6\% Vertrel XF (Du Pont-Mitsui Fluorochemicals Co., Tokyo) (27) and then separated by centrifugation at $12,600 \times g$ for $10 \mathrm{~min}$. The supernatant was recentrifuged at $29,000 \mathrm{rpm}(\mathrm{RCF}$ Max $86,600 \times g)$ for $2.5 \mathrm{~h}$, and the pellet was resuspended in TN buffer. The suspension was layered onto a 20 to $50 \%$ sucrose density gradient and centrifuged at $24,000 \mathrm{rpm}$ $($ RCF Max $102,300 \times g$ ) for $1 \mathrm{~h}$. Fractions between 35 and $25 \%$ were separately collected in 500- $\mu \mathrm{l}$ aliquots, and the relative amount of virus in each fraction was estimated based on the amount of dsRNA, using the RNaid KIT (BIO101, Vista, CA). The two to three fractions containing the highest concentration of dsRNA were mixed, diluted with TN buffer, and centrifuged at $34,000 \mathrm{rpm}(\mathrm{RCF}$ Max $104,000 \times g)$ for $1 \mathrm{~h}$. The resultant pellet was resuspended in $\mathrm{TN} 2$ buffer $(10 \mathrm{mM}$ Tris/HCl [pH7.4], $150 \mathrm{mM} \mathrm{NaCl}$, and $10 \mathrm{mM} \mathrm{MgCl}_{2}$ ).

Protoplast transfection. Protoplasts of Diaporthe sp. were prepared following the procedure of Turgeon et al. (42) with some modifications. Briefly, mycelia were washed with $0.7 \mathrm{M} \mathrm{NaCl}$, then suspended in 1\% lysing enzyme solution (L2265; SigmaAldrich, St. Louis) with $0.7 \mathrm{M} \mathrm{NaCl}$. The suspension was gently shaken at $30^{\circ} \mathrm{C}$ for $2 \mathrm{~h}$. Released protoplasts were filtered through double-layered gauze, then through a stainless, 45- $\mu \mathrm{m}$ mesh. Protoplasts were pelleted by centrifugation at $2,000 \times g$ for $10 \mathrm{~min}$, washed twice with $0.7 \mathrm{M} \mathrm{NaCl}$, and adjusted to a final concentration of $10^{8}$ protoplasts $/ \mathrm{ml}$ in SMC (1.2 M sorbitol, $10 \mathrm{mM}$ MOPS [pH 7.0], and $10 \mathrm{mM} \mathrm{CaCl}_{2}$ ).

Protoplasts of $C$. parasitica, V. ceratosperma, and G. cingulata were prepared according to Churchill et al. (6) with some modifications. Briefly, mycelia were washed with $0.6 \mathrm{M} \mathrm{MgSO}_{4}$. An enzyme solution containing $1 \%$ lysing enzyme, $1 \%$ Driselase (Kyowa Hakko Kogyo, Tokyo), and $0.1 \%$ chitinase (L6137; Sigma-Aldrich) in OM buffer (1.2 $\mathrm{M} \mathrm{MgSO}_{4}$ and $10 \mathrm{mM}$ sodium phosphate $[\mathrm{pH} 5.8]$ ) was used for $C$. parasitica and $V$. ceratosperma, and another enzyme solution containing $2 \%$ lysing enzyme, $0.5 \%$ Kitalase (Wako, Osaka, Japan), and 1\% Usukizyme (Wako) was used for G. ciugulata. Mycelial suspensions were shaken gently at $30^{\circ} \mathrm{C}$ for $2 \mathrm{~h}$. Aliquots of these suspensions were gently overlaid with TP buffer $(10 \mathrm{mM}$ Tris- $\mathrm{HCl}$ [pH5.8], including $0.4 \mathrm{M}$ sorbitol for $C$. parasitica and $V$. ceratosperma or $0.6 \mathrm{M}$ sorbitol for $G$. cingulata) and centrifuged at $1,500 \times g$ for $10 \mathrm{~min}$. Protoplasts floating at the interface were collected using a 
Pasteur pipette and diluted with $1 \mathrm{M}$ sorbitol for $C$. parasitica and $V$. ceratosperma or $1.2 \mathrm{M}$ sorbitol for $G$. cingulata. The protoplasts were washed twice by centrifugation at 3,000 $\times g$ for $5 \mathrm{~min}$ with $1 \mathrm{M}$ sorbitol, and the pellet was suspended in $1 \mathrm{M}$ STC (1 M sorbitol, $10 \mathrm{mM}$ Tris- $\mathrm{HCl}$ [pH 7.5], and $10 \mathrm{mM} \mathrm{CaCl}_{2}$ ) for $C$. parasitica and V. ceratosperma or $1.2 \mathrm{M}$ STC (1.2 M sorbitol, $10 \mathrm{mM}$ Tris- $\mathrm{HCl}$ [pH 7.5], and $10 \mathrm{mM} \mathrm{CaCl}_{2}$ ) for $G$. cingulata to achieve a final concentration of $10^{8}$ protoplasts $/ \mathrm{ml}$.

Purified virus particles of RnPV1 and MyRV3 were passed through Ultrafree-MC sterile centrifuge filter units (Millipore, Tokyo) and subjected to viral transfection assays with protoplasts. The virus particle suspensions were confirmed to be mycelium free by plating them on PDA. Viral transfection followed the standard genetic transformation procedures, with modifications
(42). Protoplasts in 50- $\mu \mathrm{l}$ aliquots were dispensed into individual Falcon 2059 tubes (Becton Dickinson), and a 5- $\mu$ l aliquot of purified virus was added to the protoplasts. Theses mixtures were kept on ice for $30 \mathrm{~min}$ and then mixed with $500 \mu \mathrm{l}$ of PEG1 solution (60\% polyethylene glycol [PEG4000], $10 \mathrm{mM}$ MOPS [pH 7.0], and $10 \mathrm{mM} \mathrm{CaCl}_{2}$ ) for Diaporthe sp. or with $500 \mu \mathrm{l}$ of PEG2 solution (60\% PEG4000, $10 \mathrm{mM}$ Tris-HCl [pH 7.5], and $10 \mathrm{mM} \mathrm{CaCl}_{2}$ ) for $C$. parasitica, V. ceratosperm, and G. cingulata. These mixtures were stirred gently and incubated for $20 \mathrm{~min}$ at room temperature. The protoplast transfection mixtures were diluted with SMC or STC before spreading on YPSA (1\% yeast extract, $1 \%$ tryptone, $0.8 \mathrm{M}$ sucrose, and $2 \%$ agar) plates. When colonies originating from protoplasts covered the entire YPSA plates, 10 mycelial plugs were taken randomly from each plate

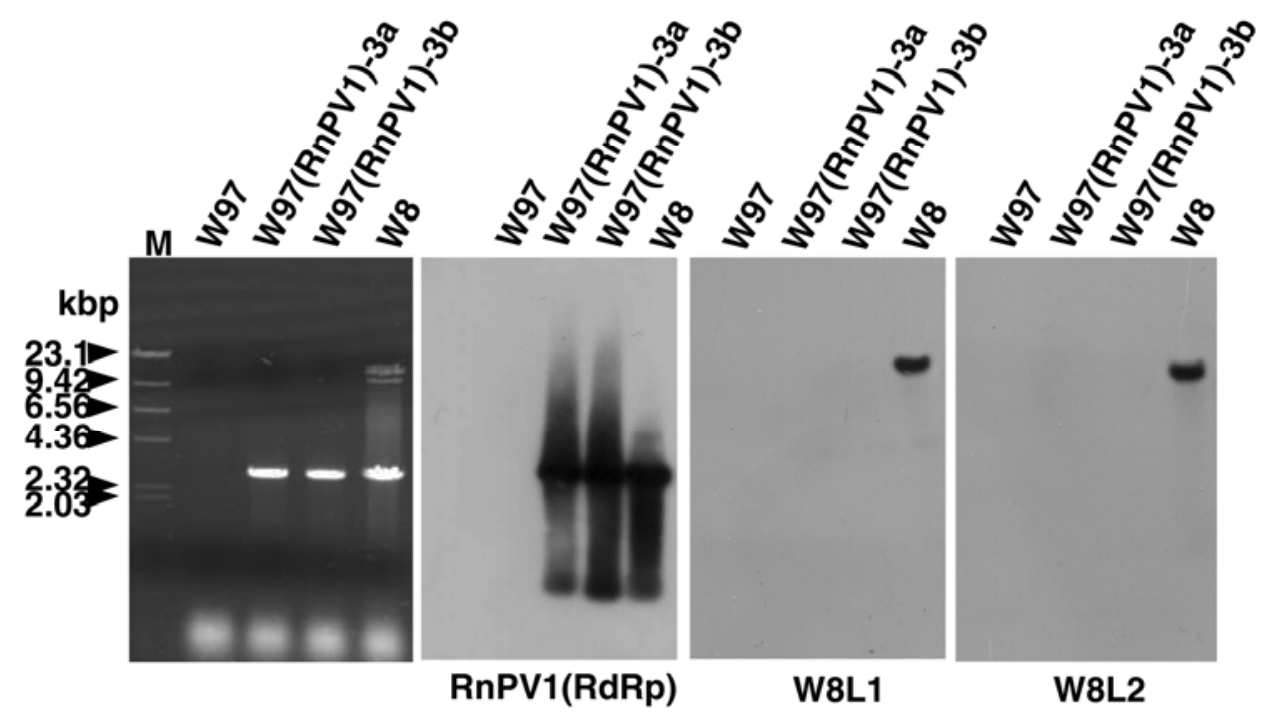

Fig. 1. Northern analysis of the transfectant W97(RnPV1)-3 (generated by artificial inoculation of Rosellinia necatrix isolate W97 with RnPV1; see details in the text) with direct labeled-L1 and L2 double-stranded (ds)RNA probes. Each lane contained dsRNA extracted from isolates shown above the panel. Two transfectants, W97(RnPV1)-3a and W97(RnPV1)-3b, were maintained independently and checked for the presence of L1 and L2 dsRNA. Lane M contained lambda DNA digested with HindIII as molecular marker. The left panel shows agarose gel with ethidium bromide staining, and other panels were hybridized membranes with the probes shown under the panels.
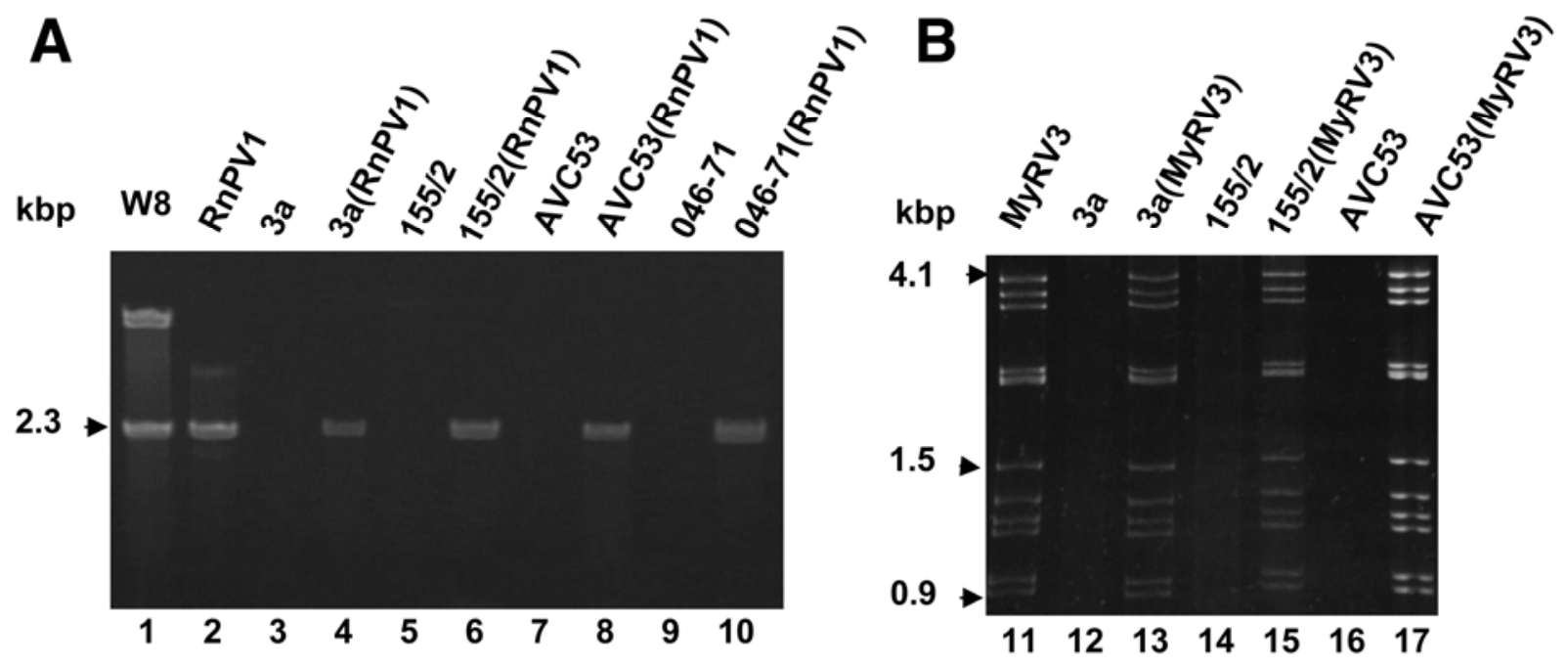

Fig. 2. A, Double-stranded (ds)RNA profile in the mycelia of wild-type and transfected strains of RnPV1-W8 was detected using agarose gel electrophoresis. Lane 1, dsRNA in the mycelia of Rosellinia necatrix isolate W8; lane 2, total RNA in virus particles of RnPV1 purified from R. necatrix strain W97(RnPV1)-3. Lanes 3-10, wild-type strains and their transfectants with RnPV1: 3a, wild-type and transfected Diaporthe sp. G-type isolate 3a; 155/2, wild-type and transfected Cryphonectria parasitica isolate 155/2; AVC53, wild-type and transfected Valsa ceratosperma isolate AVC53; 046-71, wild-type and transfected Glomerella cingulata isolate 046-71. B, MyRV3-W370 was detected using polyacrylamide gel electrophoresis. Lane 11, total RNA in virus particles of MyRV3 purified from R. necatrix isolate W370. Lanes 12-17; wild-type strains and their transfectants with MyRV3: 3a, wild-type and transfected Diaporthe sp. G-type isolate 3a; 155/2, wild-type and transfected C. parasitica isolate 155/2; AVC53, wild-type and transfected V. ceratosperma isolate AVC53. 
and subcultured on PDA. Mycelia from the subcultures were subjected to PDB culture to confirm viral infection.

Extraction of dsRNA. Extraction of dsRNA from mycelia followed the procedure of Kanematsu et al. (19). dsRNA was extracted from virus particles using the RNaid KIT (BIO101) according to the protocol of the manufacturer.

Viral transmission. Viral transmission via hyphal anastomosis was assayed using dual cultures of virus-infected (donor) and
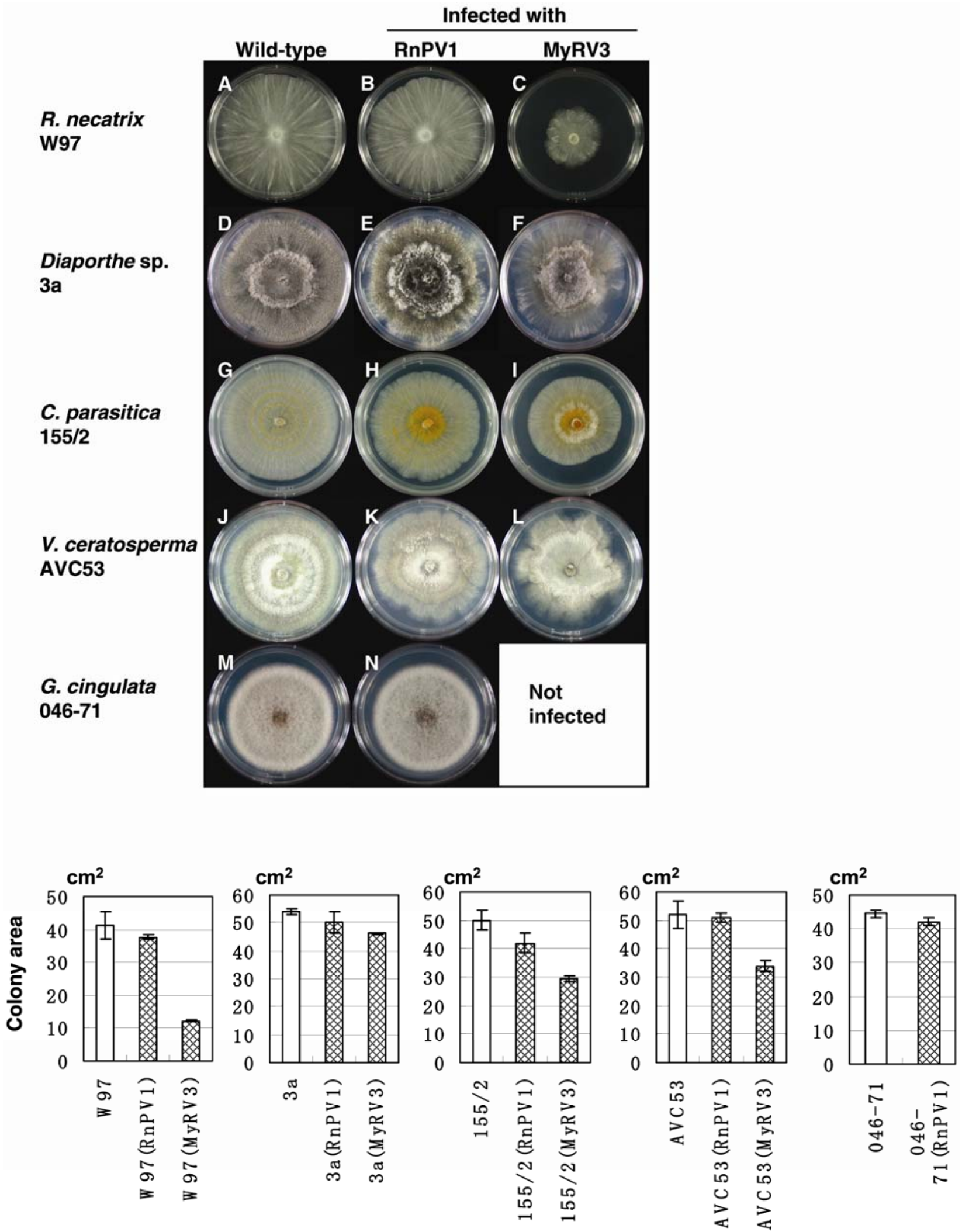

Fig. 3. Colony morphology of virus-free wild-type and virus-infected strains of A, B, and C, Rosellinia necatrix W97; D, E, and F, Diaporthe sp. 3a; G, H, and I, Cryphonectria parasitica 155/2; J, K, and L, Valsa ceratosperma AVC53; and $\mathbf{M}$ and N, Glomerella cingulata 046-71. Wild-type (A, D, G, J, and M), RnPV1W8-infected $(\mathbf{B}, \mathbf{E}, \mathbf{H}, \mathbf{K}$, and $\mathbf{N})$, and MyRV3-W370-infected $(\mathbf{C}, \mathbf{F}, \mathbf{I}$, and $\mathbf{L})$ colonies. Each strain was cultured on potato dextrose agar at $25^{\circ} \mathrm{C}$ in darkness for 6 days ( $R$. necatrix), 12-h light-and-dark cycles for 6 days (Diaporthe sp., C. parasitica, and G. cingulata), 12-h light-and-dark cycles for 4 days (V. ceratosperma). Mean colony sizes and standard errors of three to five cultures are shown in the graphs below the colony photograph. 
virus-free (recipient) strains on PDA or on oatmeal agar (OMA) (Becton Dickinson). Mycelial plugs of donor and recipient strains were placed $4 \mathrm{~cm}$ apart on a single $12-\mathrm{cm}^{2}$ dish. The dual cultures were incubated at $25^{\circ} \mathrm{C}$ in the dark. Mycelial plugs were obtained from donor and recipient colonies 12 to 13 days after dual culturing and subjected to further analysis.

Viral transmission through conidia was assayed using conidia from virus-infected strains produced on PDA after incubation at $25^{\circ} \mathrm{C}$ under 12 -h light $(\approx 4,000 \mathrm{Lux})$ and dark conditions. Conidia were collected and spread on $3 \%$ agar with a small amount of distilled water. Using a dissecting microscope and scalpel, single conidia were picked up with agar blocks and cultured for further analysis.

Horizontal and vertical transmission of RnPV1 and MyRV3 from transfectants was confirmed by dsRNA extraction from subcultures.

Virulence assay. Dormant twigs of Japanese pear and apple trees were inoculated with wild-type or virus-infected strains of Diaporthe sp. and V. ceratosperma, respectively, according to the procedure of Kanematsu et al. (20). Apple fruit were inoculated with strains of $C$. parasitica and $G$. cingulata following procedures described by Sasaki et al. (36) and incubated at $25^{\circ} \mathrm{C}$. Virus-free and virus-transfected strains of the natural host $R$. necatrix were also inoculated on apple fruit for comparison. The radii of lesions on the twigs and fruit were measured from the inoculation sites to the periphery of the lesion, and the average radiis length of lesions caused by virus-transfected strains were compared with those caused by virus-free counterpart strains using a $t$ test.

\section{RESULTS}

Transfection with virus particles of RnPV1 and MyRV3. W8 contained RnPV1, L1, and L2 dsRNAs that presumed to be of mycovirus origin (Figs. 1, left panel, and 2, lane 1), and three cycles of particle reisolation-transfection were made to remove L1 and L2 dsRNA viruses from RnPV1 to establish W97(RnPV1)$3 \mathrm{a}$ and $\mathrm{b}$. Figure 1 indicated that an RdRp probe of RnPV1 hybridized with W97(RnPV1)-3a, -3b, and W8, the original isolate infected with RnPV1. The same hybridization signals were obtained when a CP probe was used (data not shown). In contrast, hybridization signals were present exclusively in the W8 lane when the direct-labeled L1 and L2 dsRNAs were used as probes. No signal was detected in W97(RnPV1)-3a or -3b even when the membrane was exposed overnight (data not shown). These results indicated that strain W97(RnPV1)-3 was free of L1 or L2 dsRNAs.

Virus particles from purified fractions of $R$. necatrix isolates W97(RnPV1)-3 and W370 were presumed to be RnPV1 (35) and MyRV3 (42), respectively, based on their size and shape when observed by transmission electron microscopy. The absence of residual $R$. necatrix mycelia in the purified virus fractions used for transfection was confirmed by culturing these virus fractions on PDA. dsRNA genomes of the expected sizes for RnPV1 and MyRV3 were extracted from the purified virus particles (Fig. 2, lanes 2 and 11, respectively).

Electrophoretic analysis indicated that dsRNA with the same mobility as RnPV1 or MYRV3 dsRNAs was detected only in subcultured transfectants derived from regenerated mycelia and confirmed that dsRNA was absent from parental isolates of Diaporthe sp. 3a, C. parasitica 155/2, V. ceratosperma AVC53, and G. cingulata 046-71 (Fig. 2). Both RnPV1 and MyRV3 were found as separate infections in regenerated mycelia of $3 \mathrm{a}, 155 / 2$, and AVC53. We detected RnPV1 but not MyRV3 in regenerated colonies of $046-71$. We assayed $>20$ regenerated colonies $(\geq 2$ trials with 10 colonies/trial) to determine virus infection for each virus-host combination. All of the regenerated colonies of $3 \mathrm{a}$, $155 / 2$, and AVC53 had RnPV1 infections, and this virus was stable in subcultures of these fungi.
Effect of viral infection on fungal colony. Colony morphology of the virus-infected strains and virus-free parental controls grown under the same culture conditions were compared. Representative colonies and mean area of virus-free and virus-transfected strains are shown in Figure 3. Colonies of the natural host R. necatrix W97 and its transfectants with RnPV1 (this study) or MyRV3 (33) are presented for comparison. Infection with RnPV1 had little effect on colony morphology of $R$. necatrix W97, Diaporthe sp. 3a, C. parasitica $155 / 2$, and V. ceratosperma AVC53 and no effect on G. cingulata 046-71 (Fig. 3B, E, H, K, and $\mathrm{N}$ ) compared with their virus-free parental isolates (Fig. 3A, $\mathrm{D}, \mathrm{G}, \mathrm{J}$, and M). In contrast, infection with MyRV3 resulted in visible alterations in W97, 3a, 155/2, and AVC53 colony morphology. MyRV3 infection caused slow mycelial growth in 3a ( $86 \%$ of that of its virus-free parental strain based on total area), and colonies of the $3 \mathrm{a}$ transfectants showed sparse mycelial growth compared with their virus-free counterparts (Fig. 3; compare D and F). Similarly, MyRV3 infection reduced the mycelial growth of $155 / 2$ and AVC53 (60 and 65\% relative growth, respectively) relative to virus-free controls (Fig. $3 \mathrm{G}, \mathrm{I}, \mathrm{J}$, and L). MyRV3 infection resulted in irregular margins in 155/2 colonies and amorphous growth in AVC53 colonies. The intensity of orange pigmentation of the $155 / 2$ colony was weak under the culture condition ( $12 \mathrm{~h}$ of light at 4,000 Lux and $12 \mathrm{~h}$ of darkness). Infection with either RnPV1 or MyRV3 resulted in higher orange pigmentation in $C$. parasitica $155 / 2$ (Fig. $3 \mathrm{G}, \mathrm{H}$, and I) but neither resulted in a significant difference in conidial production (data not shown).

Viral transmission. Horizontal transmission of RnPV1 and MyRV3 from virus-transfected strains to virus-free parental counterparts was examined by dual culture on PDA. Examples of RnPV1 and MyRV3 transmission in C. parasitica 155/2 are shown in Figure 4. Mycelial plugs of virus-infected donor strains (right) and of virus-free, recipient strains (left) were placed on each PDA plate. The virus was transmitted through hyphal anastomosis and altered the colony morphology of the recipient isolate. The presence of the transmitted mycovirus was confirmed by agarose gel electrophoresis of dsRNA extracted from subcultured mycelia derived from spots 1 in the donor and 2, 3, and 4 in the recipient colonies (Fig. 4A and B). Similar results were obtained from Diaporthe sp. 3a for both viruses and from $G$. cingulata 046-71 for RnPV1 only (data not shown). In the case of V. ceratosperma AVC53, neither RnPV1 nor MyRV3 were transmitted by dual culture on PDA; both infected and virus-free colonies showed repulsion and did not merge along the colony junction. Repulsion occurred on PDA between AVC53 and AVC53 (RnPV1 or MyRV3) and even between the same virus-free isolates (i.e., AVC53 and AVC53), as was the case with Leucostoma persoonii culture on PDA (1). Subsequently, AVC53 and AVC53 (RnPV1 or MyRV3) were co-cultured on OMA rather than PDA. Virus-free recipient and virus-transfected donor colonies merged well at the colony junction on OMA, and the transmission of RnPV1 and MyRV3 was confirmed by morphological change of the recipient colony and extracted dsRNA from subcultures of the recipients (data not shown).

We also examined viral transmission through conidia (Table 1). RnPV1 was rarely transmitted to single-conidial isolates $(<5 \%)$ in C. parasitica $155 / 2$ or G. cingulata 046-71, and was not transmitted in Diaporthe sp. G-type 3a. Transmission of MyRV3 through conidia was not detected in any single-conidial isolate. We could not check the transmission rate through conidia in $V$. ceratosperma because, in this species, sporulation on agar media is difficult, as reported previously (36). We further analyzed the stability of RnPV1 transmission in one case of an RnPV1-infected single-conidial isolate of $C$. parasitica $155 / 2$ using secondary single-conidial isolation. All 30 secondary, single-conidial isolates from the RnPV1-positive 155/2 primary, single-conidial isolate lost viral dsRNA through conidiation. 
Virulence assay. Diaporthe sp. G-type and V. ceratosperma are canker pathogens of Japanese pear and apple trees, respectively. Therefore, we inoculated detached twigs of Japanese pear and apple trees with mycelial plugs of the virus-transfected Diaporthe sp. and V. ceratosperma, respectively. G. cingulata causes fruit rot of apple; therefore, we inoculated apple fruit with G. cingulata and $C$. parasitica, for which an established experimental virulence evaluation in apples exists (14).

The results of virulence assays of fungal strains transfected with RnPV1 and MyRV3 are shown in Figure 5. Infection with MyRV3 significantly reduced the virulence of all tested strains$R$. necatrix W97, Diaporthe sp. 3a, C. parasitica 155/2, and V. ceratosperma AVC53-compared with the respective virus-free strains ( $t$ test; $P<0.01$ ) (Fig. 5B). In contrast, the effects of infection with RnPV1 on fungal virulence were not obvious (Fig. 5A). RnPV1-transfected 155/2 had lesions of slightly reduced length relative to the virus-free controls, $(0.01<P<0.05)$, and the virulence of the virus-free W97, 3a, and AVC 53 strains were not significantly different from the transfectants $(P>0.05)$.

\section{DISCUSSION}

$R$. necatrix belongs to class Sordariomycetes, subclass Xylariomycetidae. Class Sordariomycetes comprises three subclasses: Sordariomycetidae (Diaporthe sp., C. parasitica, and V. ceratosperma), Hypocreomycetidae ( $G$. cingulata), and Xylariomycetidae (38). We confirmed that artificial inoculation with RnPV1-W8 and MyRV3-RnW370 extends the experimental host range of these viruses beyond subclass Xylariomycetidae to other subclasses in Sordariomycetes. This study showed that artificial inoculation of virus particles by PEG-mediated protoplast transfection paves the way for expanding the experimental host ranges of mycoviruses, despite the absence of an extracellular route for mycovirus transmission $(11,25)$. Compared with animals and plants, fungi can readily regenerate from protoplasts or single cells. If a mycovirus is introduced to and replicates in the cytoplasm of a fungal protoplast, the virus is very likely to propagate and distribute throughout the new host, as demonstrated in the hyphal anastomosis transmission tests of RnPV1 and MyRV3 (Fig. 4). Our data suggest that the septal pores of fungi (3) are large enough to allow the passage of a mycovirus and mediate intramycelial transmission.

This study demonstrated that MyRV3 infection caused colony alteration and, importantly, hypovirulence in a broad range of Sordariomycetous phytopathogenic fungi, including pathogens of serious canker on fruit trees (Diaporthe sp., C. parasitica, and $V$. ceratosperma). The observed differences in the effects of RnPV1 and MyRV3 infection on the virulence of Sordariomycetous fungi were not unexpected, considering that RnPV1 is associated with cryptic infection in R. necatrix (34), and mycoreoviruses MyRV1, MyRV2, and MyRV3 attenuate the virulence of their natural hosts, C. parasitica for MyRV1 (14) and MyRV2 (10) and $R$. necatrix for MyRV3 (33). Despite its importance as a fruit tree pathogen, the mechanism underlying $R$. necatrix pathogenesis is

TABLE 1. Transmission through conidia in virus-infected strains of Diaporthe sp. G-type, Cryphonectria parasitica, and Glomerella cingulata

\begin{tabular}{llcc}
\hline & & \multicolumn{2}{c}{ No. of single-conidial isolates } \\
\cline { 3 - 4 } Species & Strain & Total & Virus infected \\
\hline Diaporthe G-type & 3a (RnPV1) & 58 & 0 \\
& 3a (MyRV3) & 59 & 0 \\
C. parasitica & 155/2(RnPV1) & 20 & $1^{\mathrm{b}}$ \\
G. cingulata & 155/2 (MyRV3) & 20 & 0 \\
\hline
\end{tabular}

a All the single-conidial isolates derived from virus-infected strains were checked for the presence of viral double-stranded RNA genome by agarose gel electrophoresis.

b We further analyzed this isolate to obtain 30 single-conidial subcultures, all of which proved to be virus free.
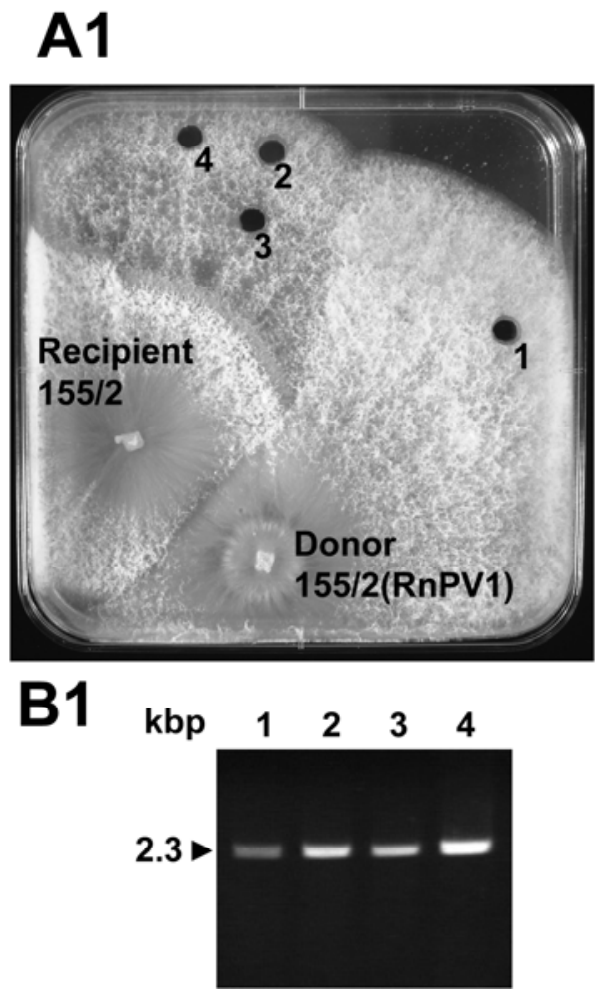

A2
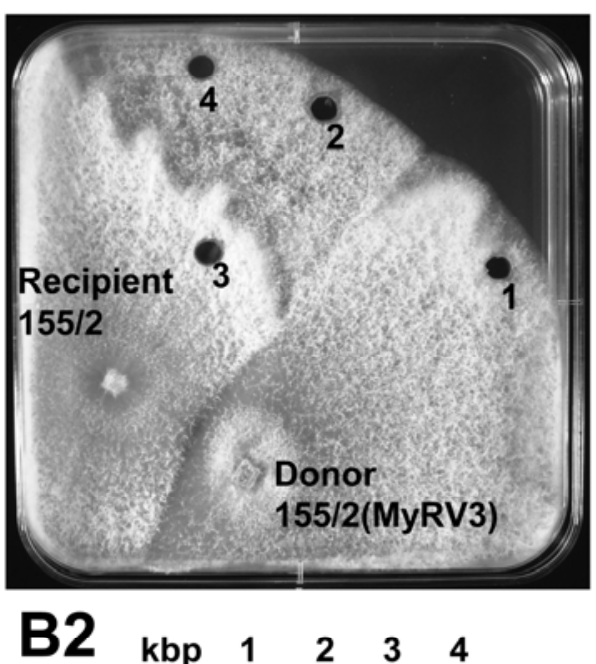

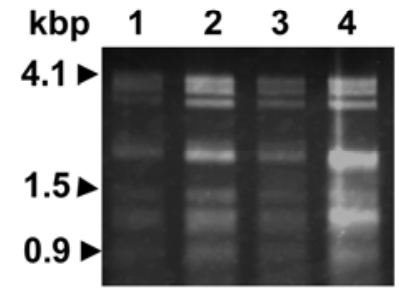

Fig. 4. Transmission of RnPV1 or MyRV3 via hyphal anastomosis. A, Mycelial plugs of recipient wild-type isolates (155/2) and donor virus-infected strains (A1, 155/2(RnPV1) and A2, 155/2(MyRV3)) were cultured on potato dextrose agar for 13 days. B, Double-stranded (ds)RNA was extracted from subcultures of the mycelial plugs obtained from the numbered positions in A1 and A2. The presence of the transmitted mycovirus was confirmed by agarose gel electrophoresis of the extracted dsRNA (B1, RnPV1 and B2, MyRV3). 
still poorly understood. Hypovirulence of both $C$. parasitica and $R$. necatrix associated with MyRV3 infection may build on the earlier contributions to the understanding of $C$. parasitica pathogenesis $(7,17)$ in future studies of $R$. necatrix pathogenesis.

RnPV1 infection is very stable in mycelia of $R$. necatrix (35). An attempt to prepare virus-free strains by hyphal tip isolation was unsuccessful, because all derivative isolates harbored RnPV1. Conidia of $R$. necatrix seldom germinated and the germinated conidia never develop into mycelia, at least under laboratory conditions (30). Therefore, we could not survey the transmission frequency of RnPV1 through the conidia of $R$. necatrix. The high stability of RnPV1 infection in R. necatrix that we observed is consistent with the general view that partitivirus infections are stable in fungal mycelia with high transmission rates (90 to $100 \%)$ through conidia $(12,15,41)$. In contrast, the stability of mycoreoviruses in their natural hosts varies depending on the virus strains. For example, MyRV1-Cp9B21 was stable in mycelia of $C$. parasitica, and its transfectants were also stable after repeated subcultures (14). MyRV2-CpC18 was less stable in culture than MyRV1-Cp9B21 (15). MyRV1 and MyRV2 transmitted through conidia infrequently, at rates of $<10 \%(7,39)$. MyRV3-RnW370 was unstable and showed uneven distribution in single colonies of $R$. necatrix (33). In the present study, it was rare for either RnPV1 or MyRV3 to be transmitted through the conidia of an experimental host species (Table 1). The transmission rates of RnPV1-W8 through conidia of the experimental hosts were lower than the high transmission rates observed for other partitiviruses in their natural hosts $(12,15,41)$. In our tests, it is unlikely that some somatically mutated single-conidial strains could keep RnPV1 upon repeated conidiogenesis in new hosts, because the single-conidial isolates which harbored RnPV1 did not keep RnPV1 after the next conidiation (Table 1). Novel virushost fungus interactions may be unstable and, for a mycovirus that is transmitted from its natural host to a novel host in nature, transmission through conidiation is an important milestone in the establishment of a stable, novel host-virus relationship.

Mycoviruses have no extracellular route for entry into new natural hosts; therefore, their host ranges are assumed to be limited to individuals within the same or vegetatively compatible groups. There are some reports of interspecies transmission of mycoviruses between closely related species, such as transmission of hypovirus-associated dsRNA from Sclerotinia sclerotiorum to S. minor (26) and CHV1 from C. parasitica to an unidentified Cryphonectria sp. (23). Though mycelial incompatibility responses occurred in these combinations to form barriers between the colonies, the viruses apparently had some chance to
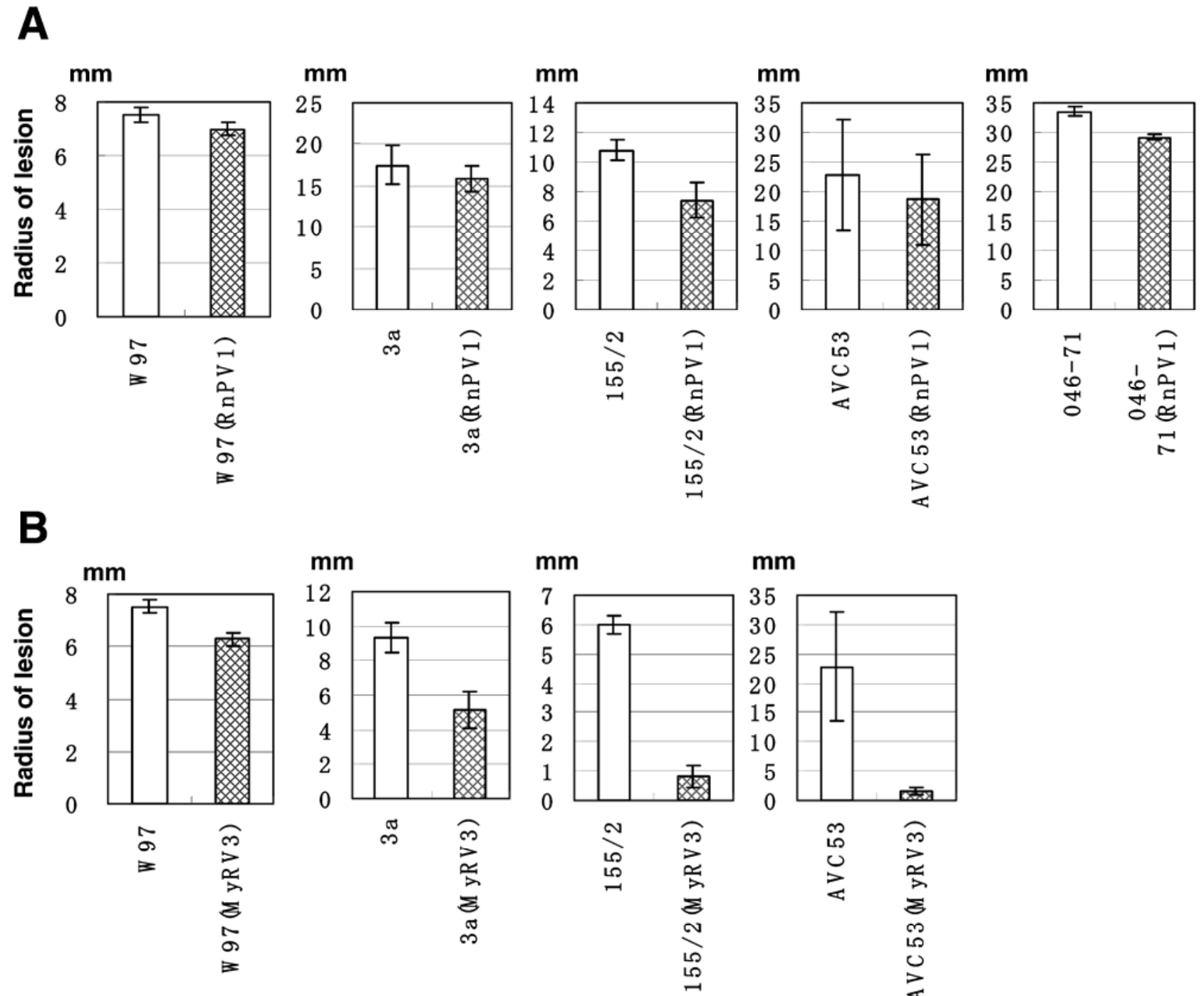

Fig. 5. Virulence of fungal strains infected with RnPV1or MyRV3. Each column indicates the average radius of the lesions cause by A, RnPV1 or B, MyRV3 infections. Lesions developed on apple fruit (14 days), Japanese pear dormant twig (13 days), apple fruit (8 days), apple dormant twig (10 days), and apple fruit (10 days) after inoculation with mycelial plugs containing virus-transfected or virus-free Rosellinia necatrix W97, Diaporthe sp. 3a, Cryphonectria parasitica 155/2, Valsa ceratosperma AVC53, and Glomerella cingulata 046-71, respectively. Each column represents five to six replications, and the error bars represent standard errors. 
transmit between colonies. The natural occurrence of conspecific mitoviruses in two distantly related fungi, $S$. homoeocarpa and Ophiostoma novo-ulmi, suggests that horizontal transmission of the progenitor mitovirus occurred in nature between these two fungi and their associations with persistent infections (8). Our study demonstrated that the mycoreovirus MyRV3 or the partitivirus RnPV1 introduced into the cells of fungi distantly related to their natural hosts may replicate in the cytoplasm of the new host and establish a stable or semistable infection.

The four new, experimentally induced hosts (Diaporthe sp. 3a, C. parasitica $155 / 2, V$. ceratosperma AVC53, and G. cingulata 046-71) have ecological niches similar to that of the natural host, $R$. necatrix. All of these fungi are deciduous fruit tree pathogens, causing diseases such as apple root rot, canker, and fruit rot. Due to their ecological proximity, natural horizontal transmission of these viruses may be probable even if these fungi are not likely to fuse with each other; however, we did not detect MyRV3 or RnPV1 transmission from $R$. necatrix to the newly identified species by dual culture (data not shown). Interspecies hyphal fusion may require drastic evolutionarily events such as gene exchange between distantly related fungi (37). Alternatively, horizontal transmission of mycoviruses may be mediated by animal vectors, such as mites and nematodes. It is noteworthy that MyRV3 is closely related to an animal coltivirus transmitted by ticks in nature $(14,40,43,44)$ and that virus particles of both RnPV1 and MyRV3 are infectious after they are taken from their host cells.

Expanding the experimental host range of mycoviruses and hypovirulence factors increases the potential to study biocontrol of fungal diseases and virus-host interactions. In addition to MyRV3, we have recently found another virus, $R$. necatrix megabirnavirus 1 , that also reduces the virulence of $R$. necatrix (5). These mycoviruses may serve as virocontrol agents. The term "virocontrol" is defined as one form of biological control that uses viruses capable of infection and compromises organisms that are pathogens of useful organisms, such as crops and livestock (13). If mycoviruses can be introduced into a wide range of phytopathogenic fungi to reduce virulence, mycoviruses should have great potential as virocontrol agents. Expanding the host range of mycoviruses may also be useful in understanding the molecular mechanisms of fungal pathogenicity. Comparison of the transcription profiles of $C$. parasitica infected with $\mathrm{CHV} 1$, MyRV1, or MyRV2 revealed significant overlap in the set of genes for which expression was affected by viral infection (7). Similarly, comparison of gene expression profiles in different host fungi following infection by a single mycovirus, such as MyRV3, might provide new insights into the mechanisms of pathogenicity of different phytopathogenic fungi.

\section{ACKNOWLEDGMENTS}

This research was supported by the program for Promotion of Basic Research Activities for Innovative Biosciences (BRAIN), and the program for Grant-in-Aid for Young Scientists (B) (KAKENHI). We thank N. Matsumoto for critical review of an early draft and N. Suzuki for helpful comments on the manuscript.

\section{LITERATURE CITED}

1. Adams, G., Hammer, S., and Proffer, T. 1990. Vegetative compatibility in Leucostoma persoonii. Phytopathology 80:287-291.

2. Arakawa, M., Nakamura, H., Uetake, Y., and Matsumoto, N. 2002. Presence and distribution of double-stranded RNA elements in the white root rot fungus Rosellinia necatrix. Mycoscience 43:21-26.

3. Bracker, C. E. 1967. Ultrastructure of fungi. Annu. Rev. Phytopathol. 5:343-372.

4. Chen, B., Chen, C.-H., Bowman, B. H., and Nuss, D. L. 1996. Phenotypic changes associated with wild-type and mutant hypovirus RNA transfection of plant pathogenic fungi phylogenetically related to Cryphonectria parasitica. Phytopathology 86:301-310.
5. Chiba, S., Salaipeth, L., Lin, Y.-H., Sasaki, A., Kanematsu, S., and Suzuki, N. 2009. A novel bipartite double-stranded RNA mycovirus from the white root rot fungus Rosellinia necatrix: molecular and biological characterization, taxonomic considerations, and potential for biological control. J. Virol. 83:12801-12812.

6. Churchill, A. C. L., Ciuffetti, L. M., Hansen, D. R., van Etten, H. D., and van Alfen, N. K. 1990. Transformation of the fungal pathogen Cryphonectria parasitica with a variety of heterologous plasmids. Curr. Genet. 17:25-31.

7. Deng, F., Allen, T. D., Hillman, B. I., and Nuss, D. L. 2007. Comparative analysis of alterations in host phenotype and transcript accumulation following hypovirus and mycoreovirus infections of the chestnut blight fungus Cryphonectria parasitica. Eukaryot. Cell 6:1286-1298.

8. Deng, F., Xu, R., and Boland., G. J. 2003. Hypovirulence -associated double-stranded RNA from Sclerotinia homoeocarpa is conspecific with Ophiostoma novo-ulmi mitovirus 3a-Ld. Phytopathology 93:1407-1414.

9. Eguchi, N., Kondo, K., and Yamagishi., N. 2009. Bait twig method for soil detection of Rosellinia necatrix, causal agent of white root rot of Japanese pear and apple, at an early stage of tree infection. J. Gen. Plant Pathol. 75:325-330.

10. Enebak, S. A., Hillman, B. I., and MacDonald, W. L. 1994. A hypovirulent isolate of Cryphonectria parasitica with multiple, genetically unique dsRNA segments. Mol. Plant-Microbe Interact. 7:590-595.

11. Ghabrial, S. A. 1998. Origin, adaptation and evolutionary pathways of fungal viruses. Virus Genes 16:119-131.

12. Ghabrial, S. A., Ochoa, W. F., Baker, T. S., and Nibert, M. L. 2008. Partitiviruses: general features. Pages 68-75 in: Encyclopedia of Virology, vol. 5. B. M. J. Mahy and V. Regenmortel, eds. Elsevier, Oxford.

13. Ghabrial, S. A., and Suzuki, N. 2009. Viruses of plant pathogenic fungi. Annu. Rev. Phytopathol. 47:353-384.

14. Hillman, B. I., Supyrani, S., Kondo, H., and Suzuki, N. 2004. A reovirus of the fungus Cryphonectria parasitica that is infectious as particles and related to the Coltivirus genus of animal pathogens. J. Virol. 78:892-898.

15. Hillman, B. I., and Suzuki, N. 2004. Viruses of the chestnut blight fungus, Cryphonectria parasitica. Adv. Virus Res. 63:423-472.

16. Ikeda, K., Nakamura, H., Arakawa, M., and Matsumoto, N. 2004. Diversity and vertical transmission of double-stranded RNA elements in root rot pathogens of trees, Helicobasidium mompa and Rosellinia necatrix. Mycol. Res. 108:626-634.

17. Jacob-Wilk, Turina, D., M., Kazmierczak, P., and Van Alfen, N. K. 2009. Silencing of Kex2 significantly diminishes the virulence of Cryphonectria parasitica. Mol. Plant-Microbe Interact. 22:211-221.

18. Kanadani, G., Date, H., and Nasu, H. 1998. Effect of Fluazinam soildrench on white root rot of grapevine. Ann. Phytopathol. Soc. Jpn. 64:139-141. (In Japanese)

19. Kanematsu, S., Arakawa, M., Oikawa, Y., Onoue, M., Osaki, H., Nakamura, H., Ikeda, K., Kuga-Uetake, Y., Nitta, H., Sasaki, A., Suzaki, K., Yoshida, K., and Matsumoto, N. 2004. A reovirus cases hypovirulence of Rosellinia necatrix. Phytopathology 94:561-568.

20. Kanematsu, S., Kobayashi, T., Kudo, A., and Ohtsu, Y. 1999. Conidial morphology, pathogenicity and culture characteristics of Phomopsis isolates from peach, Japanese pear and apple in Japan. Ann. Phytopathol. Soc. Jpn. 65:264-273.

21. Kanematsu, S., Minaka, N., Kobayashi, T., Kudo, A., and Ohtsu, Y. 2000. Molecular phylogenetic analysis of ribosomal DNA internal transcribed spacers and mating tests in Phomopsis isolates from fruit trees. J. Gen. Plant Pathol. 66:191-201.

22. Leach, J., Lang, B. R., and Yoder, O. C. 1982. Methods for selection of mutants and in vitro culture of Cochliobolus heterostrophus. J. Gen. Microbiol. 128:1719-1729.

23. Liu, Y.-C., Linder-Basso, D., Hillman, B. I., Kaneko, S., and Milgroom, M. G. 2003. Evidence for interspecies transmission of viruses in natural populations of filamentous fungi in the genus Cryphonectria. Mol. Ecol. $12: 1619-1628$

24. Matsumoto, N. 1998. Biological control of root diseases with dsRNA based on population structure of pathogens. JARQ 32:31-35.

25. McCabe, P. M., Pfeiffer, P., and van Alfen, N. K. 1999. The influence of dsRNA viruses on the biology of plant pathogenic fungi. Trends Microbiol. 7:377-381.

26. Melzer, M. S., Ikeda, S. S., and Boland, G. J. 2002. Interspecific transmission of double-stranded RNA and Hypovirulence from Sclerotinia sclerotiorum to $S$. minor. Phytopathology 92:780-784.

27. Mendez, I. I., Hermann, L. L., Hazelton, P. R., and Coombs, K. M. 2000. A comparative analysis of freon substitutes in the purification of reovirus and calicivirus. J. Virol. Methods 90:59-67.

28. Mertens, P. P. C., Hillman, B. I., and Suzuki, N. 2005. Genus Mycoreovirs. Pages 597-601 in: Virus Taxonomy: Eighth Report of the International Committee on Taxonomy of Viruses. C. M. Fauquet, M. A. Mayo, J. Maniloff, U. Desselberger, and L. A. Ball, eds. Elsevier, San Diego, CA. 
29. Montanie, H., Bossy, J. P., and Bonami, J. R. 1993. Morphological and genomic characterization of two reoviruses ( $\mathrm{P}$ and W2) pathogenic for marine crustaceans: do they constitute a novel genus of the Reoviridae family? J. Gen. Virol. 74:1555-1561.

30. Nakamura, H., Ikeda, K., Arakawa, M., and Matsumoto, N. 2002. Conidioma production of the white root rot fungus in axenic culture under near-ultraviolet light radiation. Mycoscience 43:251-254.

31. Osaki, H., Wei, C. Z., Arakawa, M., Iwanami, T., Nomura, K., Matsumoto, N., and Ohtsu, Y. 2002. Nucleotide sequences of doublestranded RNA segments from a hypovirulent strain of the white root rot fungus Rosellinia necatrix: Possibility of the first member of the Reoviridae from fungus. Virus Gene 25:101-107.

32. Pliego, C., Kanematsu, S., Ruano-Rosa, D., de Vicente, A., LopezHerrera, C., Cazorla, F. M., and Ramos, C. 2009. GFP sheds light on the infection process of avocado roots by Rosellinia necatrix. Fungal Genet. Biol. 46:137-145.

33. Sasaki, A., Kanematsu, S., Onoue, M., Oikawa, Y., Nakamura, H., and Yoshida, K. 2007. Artificial infection of Rosellinia necatrix with purified virus particles of a member of the genus Mycoreovirus reveals its uneven distribution in single colonies. Phytopathology 97:278-286.

34. Sasaki, A., Kanematsu, S., Onoue, M., Oyama, Y., and Yoshida, K. 2006. Infection of Rosellinia necatrix with purified viral particles of a member of Partitiviridae (RnPV1-W8). Arch. Virol. 151:697-707.

35. Sasaki, A., Miyanishi, M., Ozaki, K., Onoue, M., and Yoshida, K. 2005. Molecular characterization of a partitivirus from the plant pathogenic ascomycete Rosellinia necatrix. Arch. Virol. 150:1069-1083.

36. Sasaki, A., Onoue, M., Kanematsu, S., Suzaki, K., Miyanishi, M., Suzuki, N., Nuss, D. L., and Yoshida, K. 2002. Extending chestnut blight hypovirus host range within Diaporthales by biolistic delivery of viral cDNA. Mol. Plant-Microbe Interact. 15:780-789.
37. Schardl, C. L., and Craven, K. D. 2003. Interspecific hybridization in plant-associated fungi and oomycetes: a review. Mol. Ecol. 12:2861-2873.

38. Spatafora, J. W., Sung, G. H., Johnson, D., Hesse, C., O'Rourke, B., Serdani, M., Spotts, R., Lutzoni, F., Hofstetter, V., Miadlikowska, J., Reeb, V., Gueidan, C., Fraker, E., Lumbsch, T., Lucking, R., Schmitt, I., Hosaka, K. Aptroot, A., Roux, C., Miller, A. N., Geiser, D. M., Hafellner, J., Hestmark, G., Arnold, A. E., Budel, B., Rauhut, A., Hewitt, D., Untereiner, W. A., Cole, M. S., Scheidegger, C., Schultz, M., Sipman, H., and Schoch, C. L. 2006. A five-gene phylogeny of Pezizomycotina. Mycologia 98:1018-1028.

39. Sun, L., Nuss, D. L., and Suzuki, N. 2006. Synergism between a mycoreovirus and a hypovirus mediated by the papain-like protease p29 of the prototypic hypovirus CHV1-EP713. J. Gen. Virol. 87:3703-3714.

40. Suzuki, N., S. Supyani, K. Maruyama, and B. I. Hillman. 2004. Complete genome sequence of Mycoreovirus-1/Cp9B21, a member of a novel genus within the family Reoviridae, isolated from the chestnut blight fungus Cryphonectria parasitica. J. Gen. Virol. 85:3437-3448.

41. Tuomivirta, T. T., and Hantula, J. 2005. Three unrelated viruses occur in a single isolate of Gremmeniella abietina var. abietina type A. Virus Res. 110:31-39

42. Turgeon, B. G., Gabert, R. C., and Yoder, O. C. 1985. Transformation of the fungal maize pathogen Cochliobolus heterostrophus using the Aspergillus nidulans amdS gene. Mol. Gen. Genet. 201:450-453.

43. Wei, C. Z., Osaki, H., Iwanami, T., Matsumoto, N., and Ohtsu, Y. 2003. Molecular characterization of dsRNA segments 2 and 5 and electron microscopy of a novel reovirus from a hypovirulent isolate, W370, of the plant pathogen Rosellinia necatrix. J. Gen. Virol. 84:2431-2437.

44. Wei, C. Z., Osaki, H., Iwanami, T., Matsumoto, N., and Ohtsu, Y. 2004. Complete nucleotide sequences of genome segments 1 and 3 of Rosellinia anti-rot virus in the family Reoviridae. Arch. Virol. 149:773-777. 